\title{
Abundância e riqueza de espécies de Heteroptera (Hemiptera) do Parque Estadual do Turvo, sul do Brasil: Pentatomoidea
}

\author{
Letícia Santos Schmidt ${ }^{1,2} \&$ Aline Barcellos ${ }^{1}$
}

1. Museu de Ciências Naturais, Fundação Zoobotânica do Rio Grande do Sul, Rua Dr. Salvador França, 1427, 90690-000 Porto Alegre, RS, Brasil. (leticiaschmidt@yahoo.com.br; alinebar@fzb.rs.gov.br)

2. Bolsista CNPq/PIBIC.

\begin{abstract}
Abundance and species richness of Heteroptera (Hemiptera) from Parque Estadual do Turvo, southern Brazil: Pentatomoidea. A two-year study was carried out at Parque Estadual do Turvo, Municipality of Derrubadas, southern Brazil, aiming to evaluate the composition, abundance and species richness of Pentatomoidea. Samplings were conducted in the Springs of 2003 and 2004 (October), and Autumns of 2004 and 2005 (May), using beating tray method, along two trials of the park. Sampling effort (hours X collectors) totalled 153 hours. A total of 816 individuals of Pentatomoidea were collected, distributed in 56 species/morphospecies of seven families. It was found significantly less species in the Autumn 2005 than in the other periods. There was no significant difference, however, among Springs of 2003 and 2004, and Autumn of 2004, for the same parameter. Yucumã trail presented a significantly higher estimated richness than Garcia trail. Singletons and doubletons represented $44.6 \%$ of the recorded species. Aditionally, 20 other species/morphospecies of pentatomoids were obtained qualitatively by using, besides beating tray, ligth trap and visual inspections. The occurrence of species frequently associated to crops, dominant in the different sampling periods, suggests an influence of the adjacent crops on this conservation unity. New records of species for the State of Rio Grande do Sul are added.
\end{abstract}

KEYWORDS. Cydnidae, diversity, Pentatomidae, Scutelleridae, stink bugs.

RESUMO. Um estudo de dois anos foi desenvolvido no Parque Estadual do Turvo, município de Derrubadas, sul do Brasil, com o objetivo de avaliar a composição, abundância e riqueza de espécies de Pentatomoidea. As amostragens foram efetuadas nas primaveras de 2003 e 2004 (outubro) e nos outonos de 2004 e 2005 (maio), utilizando a metodologia do guarda-chuva entomológico, ao longo de duas trilhas do Parque. O esforço amostral foi medido em número de horas de coleta X número de coletores, totalizando 153 horas de amostragem. Um total de 816 indivíduos de Pentatomoidea foram coletados, distribuídos em 56 espécies/morfoespécies, pertencentes a sete famílias. A riqueza estimada de espécies no outono de 2005 foi significativamente menor do que nos demais períodos amostrados. Não houve, entretanto, diferenças significativas entre as primaveras de 2003 e 2004 e o outono de 2004, para o mesmo parâmetro. A trilha do Yucumã apresentou uma riqueza estimada significativamente maior do que a trilha do Garcia. Espécies únicas ou representadas por dois indivíduos equivaleram a 44,6\% do total de espécies registradas. Adicionalmente, 20 outras espécies/morfoespécies de Pentatomoidea foram obtidas com amostragens qualitativas, que incluíram, além de guarda-chuva entomológico, armadilhas luminosas e inspeção visual. A presença de espécies de pentatomídeos geralmente associadas a cultivos, dominantes nos diferentes períodos, sugere uma influência das culturas adjacentes a esta unidade de conservação. Novos registros de espécies para o Estado do Rio Grande do Sul são fornecidos.

PALAVRAS-CHAVE. Cydnidae, diversidade, Pentatomidae, percevejos-do-mato, Scutelleridae.

Ainda são escassos no Brasil, e em especial na Região Sul, estudos envolvendo a fauna de heterópteros. A maior parte dos levantamentos enfoca os níveis taxonômicos de família ou superfamília, e de grupos ligados a agroecossistemas. Pentatomoidea, com distribuição mundial e dez famílias registradas para o Brasil, reúne, em sua maioria, espécies fitófagas, com um pequeno grupo (Pentatomidae, Asopinae) constituído por predadores de outros insetos. Pentatomidae, a maior família, apresenta algumas espécies reconhecidamente pragas atuais ou potenciais de culturas de interessse econômico. Assim, a maioria dos levantamentos efetuados em Pentatomoidea tem enfocado apenas pentatomídeos (Galileo et al., 1977; Gastal et al., 1981; LinK \& GraZia, 1983, 1987).

A primeira lista de pentatomídeos para o Rio Grande do Sul foi elaborada por BUCKUP (1961), reunindo dados de coleções e de literatura. GASTAL et al. (1981), na região metropolitana de Porto Alegre, e LinK \& GraZIA (1983), no centro do Rio Grande do Sul, analisaram comunidades de Pentatomidae com emprego de armadilha luminosa. Link \& GRAZIA (1987) apresentaram dados de
Pentatomidae para a região central do Estado, com a compilação do resultado de levantamentos efetuados ao longo de 11 anos, através de inspeção visual, rede-devarredura e pano-de-batida. Posteriormente, GraZia et al. (1999) listaram as espécies de Pentatomoidea registradas para o Estado de São Paulo, com base principalmente em dados de literatura. Em ecossistemas naturais, Pentatomidae foi apontada por BROwN JR. (1997), em uma revisão de insetos indicadores no monitoramento da conservação, como um dos grupos com potencial para utilização como indicadores de impacto ambiental.

O primeiro levantamento de Pentatomoidea, entre outros insetos, realizado em uma unidade de conservação no estado, foi feito por BonatTo (1984) na Estação Ecológica do Taim. Esta Estação, integrante do bioma Campos Sulinos, abrange extensas áreas de várzeas, campos secos e matas de restinga arenosa e turfosa (IBAMA, 2006). Em outros ecossistemas naturais do Rio Grande do Sul, P. R. S. Bunde (comun. pess.) realizou recentemente um levantamento da diversidade de Pentatomoidea em áreas de campo, borda e mata, na Serra do Sudeste; utilizando-se de guarda-chuva entomológico 
e rede-de-varredura, analisou aspectos como sazonalidade, abundância e diversidade deste grupo na região. A abundância e riqueza de espécies de hemípteros terrestres (subordens Auchenorrhyncha e Heteroptera) foram estudadas em duas áreas da planície costeira do Rio Grande do Sul, usando o método do guarda-chuva entomológico (A. Barcellos, dados não publicados).

Este trabalho teve como objetivo descrever a composição e estrutura da assembléia de pentatomóideos do Parque Estadual do Turvo em termos de riqueza e abundância de espécies ao longo das duas trilhas do Parque, visando futuras comparações com outros ecossistemas naturais do Rio Grande do Sul. Adicionalmente, amplia-se o registro de espécies deste grupo taxonômico para o Estado, além de prover informações para a gestão e manejo daquela unidade de conservação.

\section{MATERIAL E MÉTODOS}

O Parque Estadual do Turvo (PET) é a mais antiga e uma das maiores unidades de conservação do Rio Grande do Sul, situando-se no noroeste do Estado $\left(27^{\circ} 00^{\prime}\right.$

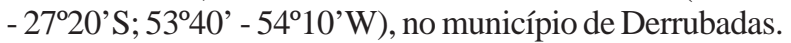
Com uma área de 17.491,40 ha, é considerado como o último reduto preservado da chamada mata pluvial subtropical do Alto Uruguai (Dias et al., 1992), que recobria boa parte desta região (Fig. 1). Esta floresta

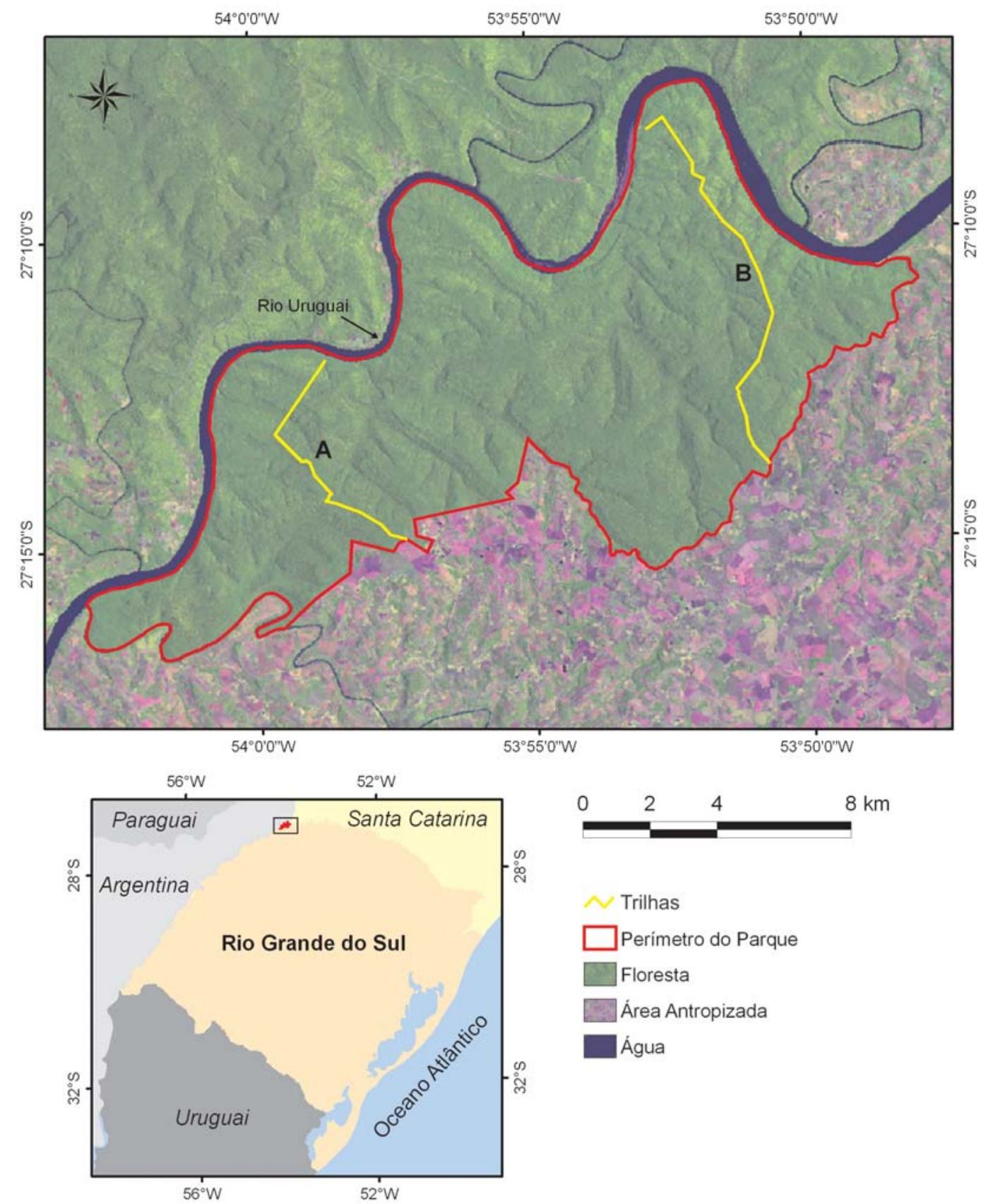

Fig. 1. Localização e imagem de satélite do Parque Estadual do Turvo, Derrubadas, RS (A, Trilha do Garcia; B, Trilha do Yucumã). 
encontra-se hoje restrita ao parque e a alguns fragmentos menores, predominando, no entorno, extensas áreas de cultivo de soja e trigo em sistema de sucessão de cultura, além de outras áreas com plantio de milho. O clima, segundo a classificação de Köppen, é do tipo Cfa, subtropical temperado úmido, com nevoeiros freqüentes, índices médios anuais de precipitação pluviométrica de até $1.900 \mathrm{~mm}$ anuais e temperaturas com variação, em média, entre $6,6{ }^{\circ} \mathrm{C}$ e $37,4{ }^{\circ} \mathrm{C}$ (VASCONCELlos et al., 1992).

As amostragens foram realizadas em quatro expedições ao PET, cada uma com duração de cinco dias, respectivamente em outubro de 2003, maio de 2004, outubro de 2004 e maio de 2005 . Nestas expedições, foram amostradas com guarda-chuva entomológico, sempre pelos mesmos três coletores, duas trilhas: a do Garcia e a do Yucumã, sendo esta última a única aberta à visitação pública. Em ambas as trilhas ocorre trânsito de veículos, porém na do Yucumã o tráfego é mais intenso, devido ao acesso de visitantes. O esforço total empregado na amostragem quantitativa foi de 153 horas, das quais 78 para a trilha do Yucumã e 75 para a do Garcia. O esforço nos diferentes períodos de amostragem totalizou: primavera/2003, 41 horas; outono/2004, 28 horas; primavera/2004, 33 horas e outono/2005, 51 horas. Adicionalmente, foram efetuadas coletas qualitativas complementares, visando ampliar a representatividade de espécies no estudo. Essas incluíram, além de coletas aleatórias com guarda-chuva entomológico, sem padronização amostral, outras técnicas, como coleta manual (total de 39 horas) e armadilha luminosa modelo Luiz de Queiroz (Silveira Neto \& Silveira, 1969) por 12 horas (uma noite).

Somente indivíduos adultos foram considerados e coletados, uma vez que imaturos são de difícil identificação. O material foi catalogado e incorporado à coleção de hemípteros terrestres do Museu de Ciências Naturais, Fundação Zoobotânica do Rio Grande do Sul. As identificações foram feitas através de comparação com material previamente identificado nas coleções científicas e/ou literatura (por exemplo, ROLSTON et al., 1980; B ARCELLOS \& GRAZIA, 1998; FORTES \& Grazia, 2005). A classificação em nível de famílias, subfamílias e tribos seguiu Schuh \& Slater (1995), com exceção do status de família para Cyrtocoridae (PACKAUSKAS \& Schaefer, 1998).

Como, embora semelhante, o esforço amostral total para coleta com guarda-chuva entomológico não foi o mesmo em cada período amostral e em cada uma das trilhas, utilizou-se o cálculo de curvas de rarefação para comparação da riqueza estimada de espécies entre os quatro períodos e entre as duas trilhas amostradas (Moreno, 2001), por meio dos programas Biodiversity Pro v. 2.0 (McAleece, 2004) e Past 1.18 (Hammer \& Harper, 2004). A estrutura da assembléia de pentatomóideos presentes no Parque foi avaliada quanto à abundância relativa de cada espécie, com a construção de uma curva de distribuição de abundâncias, também através do programa Past 1.18 (HAMMER \& HARPER, 2004), incluindo o registro do número de espécies representadas por apenas um (singletons) ou dois indivíduos (doubletons).

\section{RESULTADOS}

$\mathrm{Na}$ amostragem quantitativa com guarda-chuva entomológico, foram obtidos 816 indivíduos de 56 espécies de Pentatomoidea, 40 das quais pertencentes a Pentatomidae (Tab. I). Esta família deteve a maior abundância e também a maior riqueza de espécies. Dinidoridae, Megarididae e Tessaratomidae foram representadas nas amostragens por uma única espécie.

O número de espécies representadas por somente um (singletons) ou dois indivíduos (doubletons), respectivamente, foi de 16 e nove espécies. A proporção destas espécies foi de $44,6 \%$ em relação às demais registradas na amostragem quantitativa.

Mayrinia curvidens (Mayr, 1864) (Pentatomidae, Pentatominae) foi a mais abundante no PET, com 26,2\% exemplares coletados, ocorrendo apenas nas amostragens de outono, seguida pelos também pentatomíneos Euschistus (E.) heros (Fabricius, 1798), com 16,9\% indivíduos, e Mormidea ypsilon (Linnaeus, 1758), com $8,7 \%$ (Fig. 2), estas últimas ocorrendo nas quatro ocasiões de amostragem (Tab. I). A espécie dominante nas primaveras de 2003 e 2004 foi E. (E.) heros $(34,8 \%$ e $23 \%$, respectivamente), seguida, em 2003, por Galgupha (Euryscytus) cf. contra McAtee \& Malloch, 1933 (14,4\%) e Euschistus (Lycipta) cornutus Dallas, 1851 (8,1\%) e, em 2004, por Galgupha (Gyrocnemis) differentialis McAtee \& Malloch, $1928(11,5 \%)$ e novamente $E$. $(L$. cornutus $(8,8 \%)$. No outono de 2004 , a espécie mais abundante foi M. ypsilon (Linnaeus, 1758), com 32,4\%. No outono de 2005 houve uma alta dominância de $M$. curvidens dentre todos os pentatomóideos coletados durante esta época $(63,4 \%)$. A abundância de $M$. curvidens para o Parque deve-se especialmente a este último período amostral (outono 2005), quando 96,3\% do total dos indivíduos para esta espécie foram obtidos (Tab. I).

Considerando-se cada período amostral, as relações de dominância foram similares, com aproximadamente metade das amostras constituídas por espécies dominantes. No outono de 2005, porém, houve uma menor homogeneidade em relação aos demais períodos, devido à alta dominância apresentada por M. curvidens. Quando

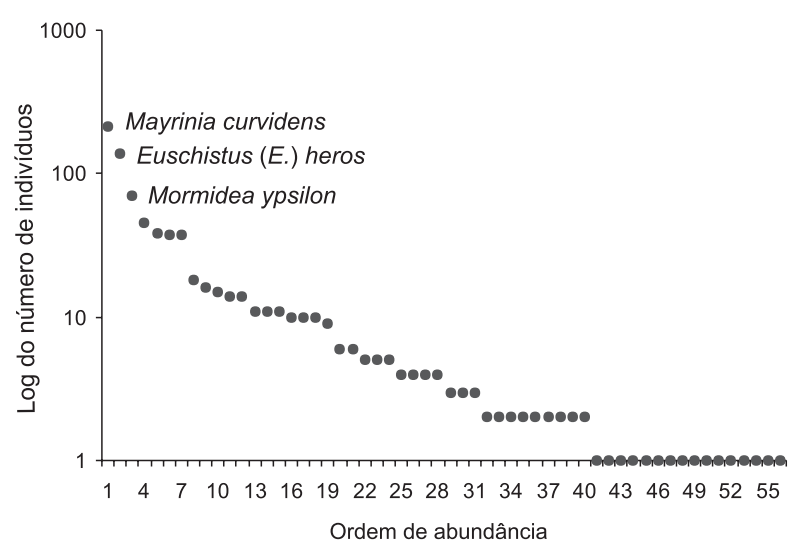

Fig. 2. Distribuição da abundância relativa de espécies de Pentatomoidea obtidas com guarda-chuva entomológico no Parque Estadual do Turvo, Derrubadas, RS, 2003-2005. 
Tabela I. Pentatomóideos do Parque Estadual do Turvo obtidos no levantamento quantitativo com guarda-chuva entomológico, Derrubadas, RS, 2003-2005 (TG, trilha do Garcia; TY, trilha do Yucumã; G, espécie exclusiva da trilha do Garcia; Y, espécie exclusiva da trilha do Yucumã).

\begin{tabular}{|c|c|c|c|c|c|c|c|c|c|}
\hline \multirow{2}{*}{ Espécies } & \multicolumn{2}{|c|}{ Prim/2003 } & \multicolumn{2}{|c|}{ Out/2004 } & \multicolumn{2}{|c|}{ Prim/2004 } & \multirow{2}{*}{\multicolumn{2}{|c|}{$\begin{array}{c}\text { Out/2005 } \\
\text { TG TY }\end{array}$}} & \multirow{2}{*}{ Total } \\
\hline & $\mathrm{TG}$ & TY & TG & TY & TG & TY & & & \\
\hline
\end{tabular}

\section{CYDNIDAE}

Galgupha (Euryscytus) cf. contra McAtee \& Malloch, 1933

Galgupha (Gyrocnemis) differentialis McAtee \& Malloch, 1928

Galgupha (Gyrocnemis) cf. diversa McAtee \& Malloch, 1933

Galgupha (Gyrocnemis) sp. 4

Cyrtomenus (Syllobus) emarginatus Stål, 1862 Y

Galgupha (Gyrocnemis) novatra McAtee \& Malloch, 1933 Y

CYRTOCORIDAE

Cyrtocoris egeris Packauskas \& Schaefer, 1998 Y

Cyrtocoris gibbus (Fabricius, 1803) ${ }^{\mathrm{Y}}$

DINIDORIDAE

Dinidor mactabilis Perty, $1833^{\mathrm{Y}}$

MEGARIDIDAE

Megaris sp. ${ }^{\mathrm{Y}}$

PENTATOMIDAE

Mayrinia curvidens (Mayr, 1864)

Euschistus (Euschistus) heros (Fabricius, 1798)

Mormidea ypsilon (Linnaeus, 1758)

Edessa sp. 4

Euschistus (Lycipta) cornutus Dallas, 1851

Serdia indistincta Fortes \& Grazia, 2005

Sibaria armata (Dallas, 1851)

Podisus distinctus (Stål, 1860)

Mormidea notulifera Stål, 1860

Dryptocephala lurida Erichson, 1848 Y

Dichelops (Neodichelops) furcatus (Fabricius, 1775)

Euschistus (Mitripus) convergens (Herrich-Schäffer, 1842)

Mormidea quinqueluteum (Lichtenstein, 1796)

Edessa sp. 2

Piezodorus guildinii (Westwood, 1837)

Platycarenus umbractulatus (Fabricius, 1803)

Alveostethus pseudopolitus (Ruckes, 1957) ${ }^{\mathrm{G}}$

Chinavia impicticornis (Stål, 1872)

Euschistus (Lycipta) picticornis Stal, 1872 Y

Thyanta (Argosoma) brasiliensis Jensen-Haarup, $1928^{\mathrm{Y}}$

Pallantia sp. nov. "A"

Proxys albopunctulatus (Palysot de Beauvois, 1805) $\mathrm{Y}$

Alveostethus latifrons (Dallas, 1851)

Cappivacius bufo Distant, 1893 Y

Edessa meditabunda (Fabricius, 1794)

Euschistus (Mitripus) paranticus Grazia, $1987^{\mathrm{G}}$

Lopadusa (Bothrocoris) quinquedentata (Spinola, 1837)

Mormidea cornicollis Stål, 1860

Supputius cinctipes $\left(\right.$ Stål,1858) ${ }^{\text {G }}$

Banasa sp. $4^{\mathrm{Y}}$

Edessa sp. 1 Y

Edessa sp. $6^{\mathrm{G}}$

Loxa virescens Amyot \& Serville, $1843^{\mathrm{G}}$

Oplomus catena (Drury, 1782) ${ }^{\mathrm{Y}}$

Pallantia macunaima Grazia, $1980^{\mathrm{G}}$

Podisus nigrispinus (Dallas, 1851) ${ }^{\mathrm{G}}$

Podisus pallipes (Dallas, 1851) Y

Stiretrus decemguttatus (Lepeletier \& Serville, 1828) ${ }^{\mathrm{G}}$

$\begin{array}{rrrrrrrrr}31 & 8 & 1 & 1 & 2 & & 1 & 2 & 46 \\ 1 & 16 & & 1 & & 13 & 5 & 1 & 37 \\ 4 & 1 & 1 & & 3 & 2 & 3 & & 14 \\ 1 & 2 & 1 & 1 & & 6 & 2 & 1 & 14 \\ & 1 & & & & & & & \end{array}$

Thyanta (Argosoma) patruelis (Stål, 1859) G

Thyanta sp. 3 Y

SCUTELLERIDAE

Symphylus sp. 2

Symphylus cf. ramivitta Walker, 1868 Y

Camirus cf. impressicollis Stål, 1862 Y

2

1

1

63
1
10
13
3
2
5

6

4

4

Lobothyreus sp. 2

Lobothyreus brasiliensis Schouteden, $1904^{\mathrm{G}}$

TESSARATOMIDAE

Piezosternum (Piezosternum) thunbergi Stål, 1860

31
4
5
9
3

8
1
7

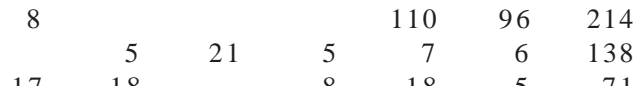
4 1 (1) 
se comparam os quatro períodos amostrados por meio de curvas de rarefação (Fig. 3), verifica-se que, para uma subamostra de 101 indivíduos, não houve diferença significativa quanto à riqueza estimada de espécies entre as primaveras de $2003\left(\mathrm{n}^{\circ}\right.$ estimado de espécies $=23$; dp $=2,30)$ e de $2004(25,9 \pm 0,95)$, e o outono de $2004(25,21 \pm$ 0,81). Entretanto, o outono de 2005 apresentou uma riqueza estimada significativamente menor do que a dos demais períodos $(17,49 \pm 2,04)$.

Ao analisarmos a riqueza das duas trilhas amostradas, na do Yucumã registraram-se 46 espécies, enquanto na trilha do Garcia foram 37. Do total de 56 espécies, dez foram exclusivas para a trilha do Garcia, 19 para a trilha do Yucumã e 27 comuns a ambas (Tab. I). Doze das espécies exclusivas para a trilha Yucumã e nove para a trilha do Garcia constituem singletons ou doubletons. Utilizando-se rarefação, observou-se que a trilha do Yucumã foi significativamente mais rica (número estimado de espécies 45,67 $\pm 0,53$ ) do que a do Garcia $(37,78 \pm 0,46)$, para uma subamostra de 401 indivíduos (Fig. 4).

O levantamento complementar com guarda-chuva entomológico, armadilha luminosa e inspeção visual adicionou 20 espécies/morfoespécies que não foram obtidas na amostragem quantitativa (Tab. II). Como exemplo, citam-se espécimes de Pachycoris torridus (Scopoli, 1772), coletados manualmente no interior de bromélias no solo, provavelmente um sítio de hibernação para esta espécie; o único exemplar de Dichelops (Dichelops) avilapiresi Grazia, 1978, capturado manualmente à noite, assim como o indivíduo de Chinavia runaspis (Dallas, 1851).

\section{DISCUSSÃO}

Apesar das diferenças quanto aos métodos de coleta empregados e/ou ao esforço amostral, pode-se comparar, de modo geral, os dados aqui apresentados com os obtidos em outras regiões do Estado. Gastal et al. (1981) identificaram 37 espécies de Pentatomidae para a região da Grande Porto Alegre e LinK \& Grazia (1983), 30 espécies para o município de Santa Maria, na região central do estado, ambos com armadilha luminosa. LiNK

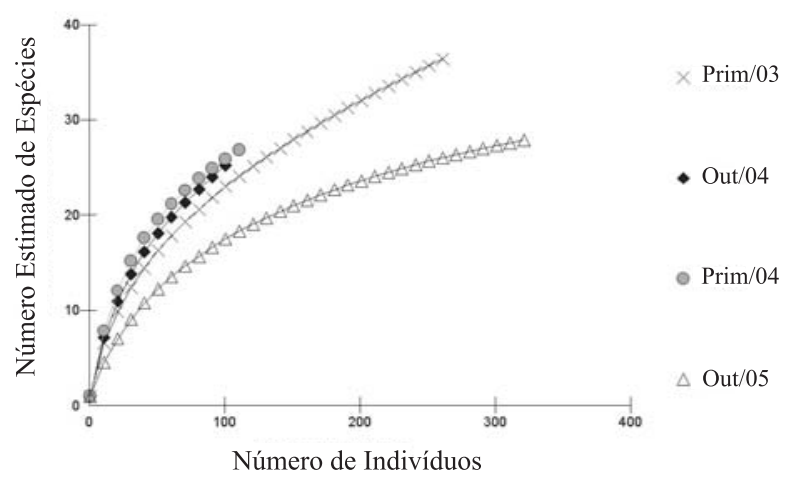

Fig. 3. Curvas de rarefação para a riqueza estimada de espécies de Pentatomoidea obtidas com guarda-chuva entomológico nas quatro ocasiões de amostragem, Parque Estadual do Turvo, Derrubadas, RS: primavera/ 2003, outono/2004, primavera/ 2004 e outono/ 2005.
\& GRAZIA (1987), para a mesma região, listaram 77 espécies de Pentatomidae, obtidas com diferentes métodos de coleta. Em áreas de borda e mata de restinga arenosa e pauldosa na planície costeira gaúcha, foram obtidos 437 indivíduos e 55 espécies de Pentatomoidea, para 171 horas de esforço amostral com guarda-chuva entomológico (A. Barcellos, dados não publicados). Para o estado como um todo, Buckup (1961) listou, com base em dados bibliográficos e de coleções, 93 espécies para Pentatomidae, das quais oito foram incluídas em subfamílias hoje consideradas famílias de Pentatomoidea (Cyrtocoridae, Scutelleridae e Tessaratomidae).

Tabela II. Pentatomóideos do Parque Estadual do Turvo obtidos exclusivamente no levantamento qualitativo, com diferentes métodos de coleta, Derrubadas, RS, 2003-2005 (CM, coleta manual; GC, guarda-chuva entomológico sem padronização; LQ, armadilha luminosa modelo Luiz de Queiroz).

\begin{tabular}{|c|c|}
\hline Espécies & Método \\
\hline \multicolumn{2}{|l|}{ PENTATOMIDAE } \\
\hline Alveostethus politus (Signoret, 1851) & GC \\
\hline Arvelius albopunctatus (De Geer, 1773) & GC \\
\hline Banasa alboapicata (Stål, 1860) & $\mathrm{GC}$ \\
\hline Banasa angulobata Thomas, 1990 & LQ \\
\hline Banasa patagiata (Berg, 1879) & GC \\
\hline Brontocoris tabidus (Signoret, 1863) & $\mathrm{GC}$ \\
\hline Chinavia runaspis (Dallas, 1851) & $\mathrm{CM}$ \\
\hline Curatia cornuta Barcellos \& Grazia, 1998 & GC \\
\hline Dichelops (Dichelops) avilapiresi Grazia, 1978 & $\mathrm{CM}$ \\
\hline Edessa sp. 3 & GC \\
\hline Janeirona bergi (Kormilev, 1956) & $\mathrm{GC}, \mathrm{CM}$ \\
\hline Macropygium reticulare (Fabricius, 1803) & GC \\
\hline Marghita similima (Grazia \& Koehler, 1983) & GC \\
\hline Mormidea hamulata Stål, 1860 & GC \\
\hline Peromatus sp. & $\mathrm{CM}$ \\
\hline Placocoris viridis Mayr, 1864 & GC \\
\hline Podisus crassimargo (Stål,1860) & GC \\
\hline Rio indistinctus Fortes \& Grazia, 2000 & GC \\
\hline \multicolumn{2}{|l|}{ SCUTELLERIDAE } \\
\hline Pachycoris torridus (Scopoli, 1772) & $\mathrm{CM}$ \\
\hline Symphylus sp. 3 & $\mathrm{CM}$ \\
\hline
\end{tabular}

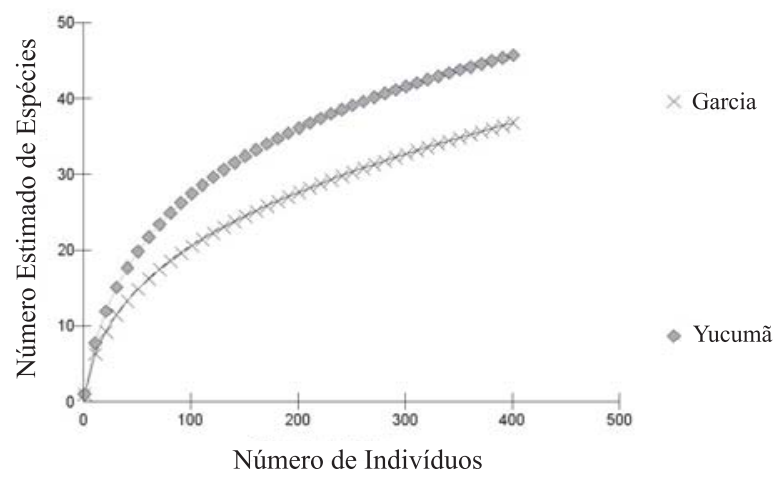

Fig. 4. Curvas de rarefação para a riqueza estimada de espécies de Pentatomoidea obtidas com guarda-chuva entomológico na trilha do Yucumã e na trilha do Garcia, Parque Estadual do Turvo, Derrubadas, RS, 2003-2005. 
A maior abundância e riqueza de Pentatomidae em relação às demais famílias já eram esperadas, visto que esta é a maior família de Pentatomoidea e a quarta mais diversa de Heteroptera, perfazendo 760 gêneros e acima de 4.000 espécies no mundo, cerca de 600 espécies no Brasil (Schun \& Slater, 1995; Grazia et al., 1999). As famílias representadas nas amostragens por uma única espécie (Dinidoridae, Megarididae e Tessaratomidae) são bem menos diversas do que Pentatomidae, e sua história natural ainda é pouco conhecida. Dinidoridae é representada na Região Neotropical somente pelo gênero Dinidor Latreille, 1829, com seis espécies descritas (GRAZIA et al., 1999). Megarididae, com apenas um gênero, Megaris Stål, 1862, possui 16 espécies, todas neotropicais (Schun \& Slater, 1995). Tessaratomidae apresenta 45 gêneros, dos quais apenas Piezosternum Amyot \& Serville, 1843 tem ocorrência na Região Neotropical, com duas espécies registradas para o Brasil (GrAZIA et al., 1999).

A quantidade de singletons e doubletons sugere que a continuidade das coletas poderia elevar o número de espécies registradas. A proporção de aproximadamente $45 \%$ dessas espécies em relação às demais pode ser decorrente de limitações do método de amostragem utilizado, por exemplo, espécies que habitam locais inacessíveis à coleta com guarda-chuva entomológico, como o dossel da mata. De fato, no PET são freqüentes árvores com altura superior a 30 m, como grápia, Apuleia leiocarpa (Vogel) MacBride (Leguminosae, Caesalpinoideae); canafístula, Peltophorum dubium (Spreng.) Taub. (Leguminosae, Caesalpinoideae); angico, Parapiptadenia rigida (Bentham) Brennan (Leguminosae, Mimosoideae) e cedro, Cedrela fissilis Vell. (Meliaceae) (IrGANG, 1980). Assim, pentatomóideos que ocupam este estrato somente seriam coletados com guarda-chuva entomológico se eventualmente abandonassem ou fossem desalojados deste microhábitat. Por outro lado, algumas dessas espécies podem ser realmente raras no PET. Para pentatomóideos da planície costeira do Rio Grande do Sul, foi obtida uma proporção de $35,2 \%$ de singletons e doubletons em relação às demais espécies registradas (A. Barcellos, dados não publicados).

Embora não se tenha amostrado as áreas de cultivo adjacentes ao PET, a dominância apresentada por $M$. curvidens, E. heros e M. ypsilon provavelmente está relacionada com uma migração destas, dos cultivos de soja (E. heros e $M$. curvidens) e trigo (M. curvidens e $M$. ypsilon) para o PET, nas respectivas entressafras (novembro a abril para o trigo e maio a outubro para a soja, aproximadamente). Estas espécies são reconhecidamente hóspedes das culturas mencionadas. Euschistus heros, conhecida como percevejo-marrom da soja, é considerada polífaga, alimentando-se, além de soja, de outras leguminosas, como tremoço, ervilha e feijoeiro (Link \& Grazia, 1987) e espécies de Solanaceae, Brassicaceae e Compositae (PANIzZI, 1997). Atualmente, E. heros é considerada uma das principais pragas da cultura da soja no Brasil (PANizzi et al., 2000). Mayrinia curvidens tem como plantas hospedeiras, conforme LiNK \& Grazia (1987), arroz e soja; até o momento, contudo, não tem sido registrada como praga importante para estas culturas. Mormidea ypsilon, de acordo com Lima (1940), alimenta-se de gramíneas cultivadas, e BuCKup (1961) cita arroz como seu item alimentar. Adultos e ninfas de $M$. ypsilon foram abundantemente coletados em Setaria spp. (Poaceae), especialmente na trilha do Garcia. A fenologia destes pentatomóideos e sua relação com plantas hospedeiras pode ser um interessante campo de investigação futura, por meio de levantamentos sistematizados com registro sazonal e simultâneo, tanto no PET como nas áreas de cultivo adjacentes. Ressaltase, por outro lado, que alguns dos pentatomídeos mais citados como pragas importantes de soja (PANIZZI et al., 2000) não foram registrados, ou estiveram representados por poucos espécimes, como Nezara viridula (Linnaeus, 1758), não registrada no PET, e Piezodorus guildinii (Westwood, 1837), com apenas cinco indivíduos.

A baixa riqueza no outono de 2005 pode ter sido resultado da intensa estiagem ocorrida no verão anterior, repetindo a situação do verão de 2003/2004. Dados da Estação Meterológica de São Luiz Gonzaga, cidade situada na mesma região do Estado, indicam, para os verões de 2003/04 e 2004/05, valores médios mensais de precipitação pluviométrica de apenas $115 \mathrm{~mm}$ e $95 \mathrm{~mm}$, respectivamente (INMET, 2006). Comparando-se estes valores com a média do verão 2003/04 (215 mm) pode-se ter idéia da severidade da seca na região, a qual, muito provavelmente, afetou a disponibilidade de alimento, sobrevivência e/ou fertilidade dos pentatomóideos. Seriam necessárias mais análises, entretanto, incluindo sazonalidade e a correlação entre as médias de precipitação pluviométrica e umidade relativa de cada estação com o número de espécies e indivíduos coletados nos mesmos períodos, para corroborar-se esta hipótese.

Em relação às duas trilhas amostradas, a maior riqueza observada no Yucumã, provavelmente, está relacionada à sua composição florística, aparentemente mais heterogênea que a da trilha do Garcia, a qual apresenta trechos mais extensos em que predomina o bambu (Merostachys skvortzovii Sendulsky, Poaceae). Conforme VAsConcellos et al. (1992) e Dias et al. (1992), a mata da trilha do Garcia seria secundária, já que teria sofrido processo de queimada em meados da década de 1980. Ressalta-se, porém, que levantamentos florísticos realizados anteriormente no Parque não foram efetuados ao longo das trilhas, e sim principalmente no interior da mata (DiAs et al., 1992; VASCONCELlos et al., 1992). Este fato impede uma conclusão a respeito do grau de heterogeneidade vegetal apresentado por ambas as trilhas.

A quantidade de espécies/morfoespécies registradas nos levantamentos qualitativos demonstra a importância de se complementar a amostragem quantitativa com coletas mais dirigidas, para fins de inventariamento de fauna. As amostragens qualitativas permitem direcionar a coleta para locais específicos, em que a probabilidade de encontrar espécimes dos grupos estudados é maior, os quais nem sempre são contemplados em procedimentos aleatórios de amostragem.

Considerando-se tanto a amostragem quantitativa quanto o levantamento qualitativo, constituem novos registros para o Rio Grande do Sul: Curatia cornuta Barcellos \& Grazia, 1998 (BARCELlos \& GRAZIA, 1998); Cyrtocoris eggeris Packauskas \& Schaefer, 1998 
(PACKAUSKAS \& Schaefer, 1998); Mormidea hamulata Stål, 1860 (Rolston, 1978), estas antes tendo Santa Catarina como limite sul brasileiro de distribuição; Cyrtomenus (Syllobus) emarginatus Stål, 1862, registrada no Brasil para Minas Gerais e São Paulo (Froeshner, 1960; Grazia et al., 1999); Pallantia macunaima Grazia, 1980, para as Regiões Sudeste, Centro-Oeste e Estado do Paraná (Grazia, 1980; Grazia \& Frey-da-Silva, 2001); Platycarenus umbractulatus (Fabricius, 1803), conhecida no Brasil da região amazônica (RUCKES, 1966); e Rio indistinctus Fortes \& Grazia, 2000, previamente com distribuição no Estado do Amazonas e Região CentroOeste (Fortes \& GraZia, 2000). Uma nova espécie de Pallantia Stål, 1862 (Pentatomidae, Pentatominae), a ser descrita oportunamente, foi registrada neste estudo, com dois indivíduos coletados na trilha do Yucumã e um na trilha do Garcia. Considerando todos os grupos de Insecta, muito provavelmente este não será o único exemplo de uma nova espécie para o Parque. A grande diversidade encontrada nesta unidade de conservação ressalta a importância do estabelecimento de ações para a efetiva preservação deste que é o último remanescente do ecossistema da mata pluvial subtropical do Alto Uruguai.

Agradecimentos. Aos colegas do grupo de pesquisa em Diversidade de Invertebrados Terrestres do MCN/FZBRS, Ricardo Ott, Ingrid Heydrich, Luciano de Azevedo Moura e Luciana R. Podgaiski, pelo companheirismo e indispensável ajuda nas amostragens. A Jan Karel Mahler Jr. e Ione de Paula (DUC-DEFAP/ RS), pelo acesso e alojamento no Parque. Aos Doutores Fernando Gertum Becker e Luiza Rodrigues Redaelli (Universidade Federal do Rio Grande do Sul), pela leitura crítica do manuscrito. Ao setor de Geoprocessamento da FZBRS, pela edição da imagem de satélite. Ao CNPq, pela concessão de bolsa à primeira autora. Ao IBAMA, pela licença de coleta e transporte de material zoológico.

\section{REFERÊNCIAS BIBLIOGRÁFICAS}

Barcellos, A. \& Grazia, J. 1998. Sobre os gêneros Curatia e Copeocoris (Heteroptera, Pentatomidae, Pentatomini). Iheringia, Série Zoologia, (85):27-46

BonatTo, S. L. 1984. Resultados preliminares do levantamento da entomofauna da Estação Ecológica do Taim, Rio Grande do Sul, Brasil. In: Semana Universitária Gaúcha de Debates Biológicos, $21^{\mathrm{a}}, 22^{\mathrm{a}}, 23,24^{\mathrm{a}}$. Anais... Porto Alegre, Universidade Federal do Rio Grande do Sul. p.46-54.

Brown, K. S., JR. 1997. Diversity, disturbance, and sustainable use of Neotropical forests: insects as indicators for conservation monitoring. Journal of Insect Conservation 1:25-42

Buckup, L. 1961. Os pentatomídeos do Estado do Rio Grande do Sul (Brasil) (Hemiptera-Heteroptera-Pentatomidae). Iheringia, Série Zoologia, (16):1-24.

Dias, L. L; Vasconcellos, J. M. de O.; Silva, C. P. da; Sobral, M. \& Benedeti, M. H. B. 1992. Levantamento florístico de uma área de mata subtropical no Parque Estadual do Turvo, Tenente Portela, RS. Revista do Instituto Florestal 4:339-346.

Fortes, N. D. F. de \& Grazia, J. 2000. Novas espécies do gênero Rio (Heteroptera, Pentatomidae). Iheringia, Série Zoologia, (88):67-102.

2005. Revisão e análise cladística de Serdia Stål (Heteroptera, Pentatomidae, Pentatomini). Revista Brasileira de Entomologia 49(3):294-339.

Froeschner, R. C. 1960. Cydnidae of the Western Hemisphere. Proceedings of the United States National Museum 3430(111):337-680.

Galileo, M. H. M.; Gastal, H. A. DE O. \& Grazia, J. 1977.
Levantamento populacional de Pentatomidae (Hemiptera) em cultura de soja (Glycine max (L.) Merr.) no município de Guaíba, Rio Grande do Sul. Revista Brasileira de Biologia 37(1):111-120

Gastal, H. A. de O.; Lanzer-de-Souza, M. E. \& Galileo, M. H. 1981. Diversidade e similaridade de comunidades de Pentatomidae (Hemiptera) capturados com armadilha luminosa na Grande Porto Alegre, Rio Grande do Sul. Iheringia, Série Zoologia, (59):5-12.

Grazia, J. 1980. Revisão do gênero Pallantia Stål, 1862 (Heteroptera, Pentatomidae). Revista Brasileira de Entomologia 24(1):15-27.

Grazia, J. \& FreY-DA-Silva, A. 2001. Descrição dos imaturos de Loxa deducta Walker e Pallantia macunaima Grazia (Heteroptera: Pentatomidae) em ligustro, Ligustrum lucidum Ait. Neotropical Entomology 30(1):73-80.

Grazia, J.; Fortes, N. F. dE \& Campos, L. A. 1999. Pentatomoidea. In: Brandão, C. R. F. \& CAncello, E. M. eds. Biodiversidade do Estado de São Paulo. São Paulo, FAPESP. v. 5, invertebrados terrestres. p.101-112.

Hammer, O. \& Harper, D. A. T. 2004. Past. Paleonthological Statistical. V. 1.18. Disponível em: <http://folk.uio.no/ ohammer/past>. Acesso em: 09.01.2004.

iBAMA. Instituto Brasileiro do Meio Ambiente e dos Recursos Naturais Renováveis. 2006. Estação Ecológica do Taim. Disponível em: <http://www.ibama.gov.br/ siucweb/>. Acesso em: 17.11.2006.

InMET. Instituto Nacional de Meteorologia. 2006. Disponível em: <http://www.inmet.gov.br>. Acesso em: 12.06.2006.

IRGANG, B. E. 1980. A mata do Alto Uruguai no Rio Grande do Sul. Ciência e Cultura 32:323-324.

Lima, A. DA C. 1940. Insetos do Brasil: Hemípteros. Rio de Janeiro, Escola Nacional de Agronomia. v.2 (série didática 3). 351p.

Link, D. \& Grazia, J. 1983. Pentatomídeos capturados em armadilha luminosa em Santa Maria, RS, Brasil. Anais da Sociedade Entomológica do Brasil 12(1):123-125.

1987. Pentatomídeos da região central do Rio Grande do Sul (Heteroptera). Anais da Sociedade Entomológica do Brasil 16(1): 116-129.

McAleece, N. 2004. Biodiversity Professional 2.0. The Natural History Museum and the Scotish Association for Marine Science. Disponível em: http://www.nhm.ac.uk/zoology/ bdpro>. Acesso em: 12.12.2004.

Moreno, C. E. 2001. Métodos para medir la biodiversidad Zaragoza, Sociedad Entomológica Aragonesa. Manuales y Tesis SEA, v. 1. 84p.

Packauskas, R. J. \& Schaefer, C. W. 1998. Revision of Cyrtocoridae (Hemiptera: Pentatomoidea). Annals of the Entomological Society of America 91(4):363-386.

Panizzi, A. R. 1997. Wild hosts of Pentatomids: ecological significance and role in their pest status on crops. Annual Review of Entomology 42:99-122.

Panizzi, A. R.; McPherson, J. E.; James, D. G.; Javahery, M. \& McPherson, R. M. 2000. Stink bugs (Pentatomidae). In: Schaefer, C. W. \& Panizzi, A. R. eds. Heteroptera of Economic Importance. New York, CRC Press. p.421-474.

Rolston, L. H. 1978. A revision of the genus Mormidea (Hemiptera: Pentatomidae). New York Entomological Society 86(3): 161-219.

Rolston, L. H.; McDonald, F. J. D. \& Thomas, D. B, JR. 1980. A conspectus of Pentatomini genera of the Western Hemisphere - part 1 (Hemiptera: Pentatomidae). Journal of the New York Entomological Society 88(2):120-132.

RUCKES, H. 1966. An analysis and a breakdown of the genus Platycarenus Fieber (Heteroptera, Pentatomidae, Discocephalinae). American Museum Novitates (2255):1-42.

Schun, R. T. \& Slater, J. A. 1995. True bugs of the World (Hemiptera: Heteroptera). Classification and Natural History. Ithaca, Cornell University. 336p.

Silveira Neto, S. \& Silveira, A. C. 1969. Armadilha luminosa modelo Luiz de Queiroz. O Solo 61(2):15-21.

Vasconcellos, J. M. de O.; Dias, L. L.; Silva, C. P. da \& Sobral, M. 1992. Fitossociologia de uma área de mata subtropical no Parque Estadual do Turvo - RS. Revista do Instituto Florestal 4:252-259 